\title{
Off-line test of the KISS gas cell
} \author{
Michiharu Wada ${ }^{d}$, Mark Huyse ${ }^{\mathrm{e}}$, Yuri Kudryavtsev ${ }^{\mathrm{e}}$, Piet Van Duppen ${ }^{\mathrm{e}}$ \\ a Institute of Particle and Nuclear Studies (IPNS), High Energy Accelerator Research Organization (KEK), Ibaraki 305-0801, Japan \\ ${ }^{\mathrm{b}}$ Seoul National University, Seoul 151 742, Republic of Korea \\ ${ }^{\mathrm{c}}$ Tsukuba University, Ibaraki 305 0006, Japan \\ ${ }^{\mathrm{d}}$ Nishina Center for Accelerator-Based Science, RIKEN, Wako, Saitama 351 0198, Japan \\ e Instituut voor Kern-en Stralingsfysica, KU Leuven, B-3001 Leuven, Belgium
}

Yoshikazu Hirayama a,*, Yutaka Watanabe ${ }^{a}$, Nobuaki Imai ${ }^{a}$, Hironobu Ishiyama ${ }^{a}$ Sun-Chan Jeong ${ }^{a}$ Hiroari Miyatake $^{\mathrm{a}}$, Michihiro Oyaizu ${ }^{\mathrm{a}}$, Yung Hee Kim ${ }^{\mathrm{b}}$, Momo Mukai ${ }^{\mathrm{c}}$, Yukari Matsuo ${ }^{\mathrm{d}}$, Tetsu Sonoda ${ }^{\mathrm{d}}$,

\section{A R T I C L E I N F O}

\section{Article history:}

Received 18 March 2013

Received in revised form 8 June 2013

Accepted 20 June 2013

Available online 4 July 2013

\section{Keywords:}

Gas cell

Laser resonance ionization

Beta-decay spectroscopy

\begin{abstract}
A B S T R A C T
The KEK Isotope Separation System (KISS) has been constructed at RIKEN to study the $\beta$-decay properties of neutron-rich isotopes with neutron numbers around $N=126$ for application to astrophysics. A key component of KISS is a gas cell filled with argon gas at a pressure of $50 \mathrm{kPa}$ to stop and collect the unstable nuclei, where the isotopes of interest will be selectively ionized using laser resonance ionization. We have performed off-line tests to study the basic properties of the gas cell and of KISS using nickel and iron filaments placed in the gas cell.
\end{abstract}

(c) 2013 Elsevier B.V. All rights reserved.

\section{Introduction}

The beta-decay properties of nuclei with $N=126$, which act as progenitors in the r-process path forming the third peak $(A \approx 195)$ in the $\mathrm{r}$-abundance element distribution, are considered critical for clearly understanding the production of heavy elements such as gold and platinum at astrophysical sites [1]. We have constructed the KEK Isotope Separation System (KISS) to produce pure low-energy beams of neutron-rich isotopes around $N=126$ and to study their beta-decay properties, which are also of interest for astrophysics [2].

We plan to produce and study ${ }^{200} \mathrm{~W},{ }^{201} \mathrm{Re},{ }^{202} \mathrm{Os}$ and ${ }^{203} \mathrm{Ir}$ ( $Z=74-77, N=126$ ) by multi-nucleon transfer (MNT) reactions [3] between an energetically stable ${ }^{136} \mathrm{Xe}$ beam at $10 \mathrm{MeV} /$ nucleon and a ${ }^{198} \mathrm{Pt}$ target. The recoil energies of nuclei with $N=126$ produced as target-like fragments are as low as $1 \mathrm{MeV} /$ nucleon and have a wide energy distribution. The emission angles for this recoil reaction calculated by the GRAZING code [4] vary widely around an average value of $65^{\circ}$ in the laboratory frame. The first results from experiments on this reaction are reported in Ref. [5].

The characteristics of the MNT reaction products make it difficult to collect nuclei with $N=126$ using an in-flight-type electromagnetic spectrometer. Therefore, we employ a gas catcher to efficiently collect all reaction products and use the laser resonance

\footnotetext{
* Corresponding author.Tel.: +81 298796060.

E-mail address: yoshikazu.hirayama@kek.jp (Y. Hirayama).
}

ionization technique to select nuclei with specific atomic numbers $Z$ from the collected nuclei, and an electromagnetic separator (ISOL) to obtain nuclei with specific mass numbers $A$. This type of gas catcher system has been developed and applied effectively to the study of nuclear structure by the Leuven group [6-11].

We use a gas cell filled with argon gas at a pressure of $50 \mathrm{kPa}$ in conjunction with a laser resonance ionization technique for the selective ionization of the isotopes of interest. In this paper we report the results of off-line tests to investigate the performance of the KISS gas cell.

\section{KISS setup}

Fig. 1 shows a schematic layout of KISS, which was constructed at the RIBF facility in RIKEN at the beginning of 2011. It consists of a laser system, a mass-separator system, three decay measurement stations and a gas-cell system.

The laser system consists of two frequency-tunable dye lasers pumped by two excimer $(\mathrm{XeCl}, 308 \mathrm{~nm}$ ) lasers and is installed in a separate room below the KISS gas cell system. A repetition rate of up to $200 \mathrm{~Hz}$ is used and the bandwidth of the dye laser is $0.15 \mathrm{~cm}^{-1}$. The frequency of the first step laser is doubled using a second harmonic generator (BBO crystal). The distance between the laser system and the gas cell is about $15 \mathrm{~m}$. In the gas cell, both lasers overlap for resonance ionization, spatially and temporally.

The mass-separator system has a QQDQQ configuration, in which $\mathrm{Q}$ and $\mathrm{D}$ denote quadrupole and dipole magnets, respec- 


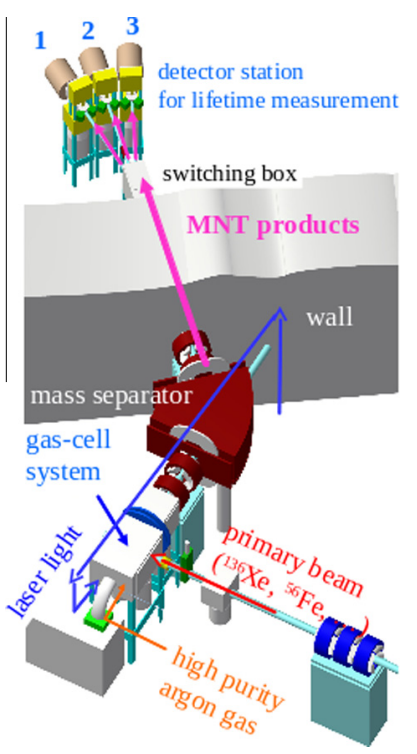

Fig. 1. Schematic layout of KISS.

tively. The deflection angle and pole gap of the D magnet are 45 degrees and $70 \mathrm{~mm}$, respectively. The expected mass resolving power is 900 [12].

Each of the three decay measurement stations has its own tape transport device for decay measurements under pulsed beams from the separator. Plastic scintillator telescopes followed by germanium gamma-ray detectors cover the implantation point in the tape transport device (Fig. 1).

The gas-cell system (Fig. 2) includes an argon gas feeding line with a gas-purification device (Monotorr Phase II 3000), a gascatcher cell, and a sextupole ion-guide (SPIG) [13]. To enable extraction of isotopes as an ion-beam with sufficiently low emittance and high efficiency, the gas-cell system employs differential pumping to reduce the pressure in the vacuum chamber from $50 \mathrm{kPa}$ to several $10^{-4} \mathrm{~Pa}$. When the ions leave the gas cell, their transit through the SPIG is supported by the gas jet, though the gas jet may cause electrical discharge between the SPIG and an extraction electrode and hence make it difficult to apply the necessary acceleration voltage (e.g. $28 \mathrm{kV}$ ). Therefore, the vacuum chamber of the gas-cell system is separated into three rooms for the differential pumping. The first, second and third rooms are pumped down by a $175 \mathrm{l} / \mathrm{s}$ screw pump, two $800 \mathrm{l} / \mathrm{s}$ turbo molecular pumps (TMPs) and a $1500 \mathrm{l} / \mathrm{s}$ TMP, respectively. The three neighboring rooms are connected by the SPIG with an aperture of $3 \mathrm{~mm}$ diameter and $200 \mathrm{~mm}$ length. The conductance between two adjacent

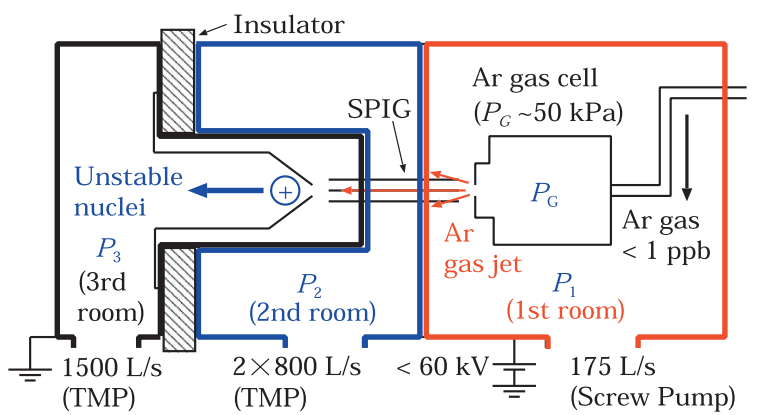

Fig. 2. Schematic view of the gas-cell system. The boundaries of the first, second and third rooms are indicated by the thick red, blue and black lines, respectively. (For interpretation of the references to colour in this figure legend, the reader is referred to the web version of this article.)
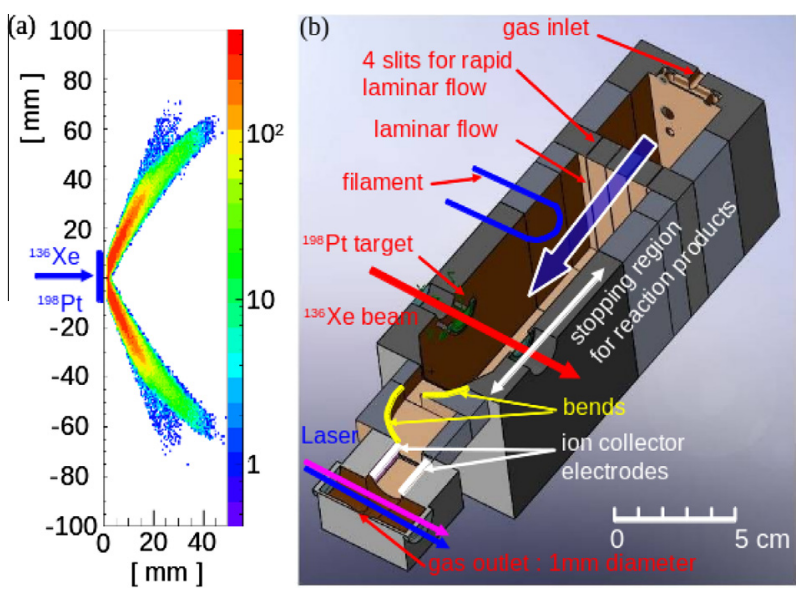

Fig. 3. (a) Stopping distribution of ${ }^{202} \mathrm{Os}$ in argon gas with a pressure of $50 \mathrm{kPa}$. (b) Schematic cross sectional view of the gas cell.

rooms was calculated to be $0.15 \mathrm{l} / \mathrm{s}$. The gas cell filled with argon gas at a pressure of $50 \mathrm{kPa}$ is placed in the first room. The conductance of the gas cell exit, which has an aperture of $1 \mathrm{~mm}$ diameter and $0.5 \mathrm{~mm}$ length, was calculated to be $0.095 \mathrm{l} / \mathrm{s}$. The second room is important for differential pumping and ensures that the vacuum conditions in the third room are sufficient for applying a high voltage to the first and second rooms (several $10^{-4} \mathrm{~Pa}$ ). Typical pressure values in the three rooms are $P_{1}=8.7 \mathrm{~Pa}, P_{2}=0.9 \mathrm{~Pa}$ and $P_{3}=3 \times 10^{-4} \mathrm{~Pa}$, for $P_{\mathrm{G}}=50 \mathrm{kPa}[14]$.

Fig. 3(a) shows the calculated stopping distribution of ${ }^{202} \mathrm{Os}$ in argon gas with a pressure of $50 \mathrm{kPa}$. Here, the energies and emission angles of the ${ }^{202}$ Os nuclei were calculated by the GRAZING code [4] and the stopping power was calculated by the SRIM2008 code [15]. The stopping distribution exhibits a wide spread. Therefore, to obtain a high efficiency (90\%), a large gas cell volume was considered, as shown in Fig. 3(b). The gas cell has four slits to generate a rapid laminar flow of argon gas. Considering the stopping distribution of ${ }^{202} \mathrm{Os}$, the transport efficiency is calculated to be $56 \%$, where the remainder is lost at the wall due to diffusion. The simulation method in the gas cell is described in Ref. [16]. In order to increase the selectivity, the ion collector electrodes, which absorb unwanted ions, were introduced.

Plasma induced by primary beam irradiation into the gas cell is believed to reduce the ionization efficiency and selectivity [8]. Based on the Leuven experimental result using a dual gas-cell [11], two bends for shadowing the ionization region and ion collector electrodes were introduced to limit the loss in efficiency and increase the selectivity. Considering the stopping distribution of MNT products and the structure of the bends, the gas cell was designed to achieve rapid and efficient transportation by laminar flow. The extraction efficiency is estimated to be about $5 \%$ for ${ }^{200} \mathrm{~W}(Z=74, N=126)$ nuclei with a half-life time of $423 \mathrm{~ms}$ predicted by the KUTY mode [17]. ${ }^{200} \mathrm{~W}$ nuclei is located most far from the stability line in the project.

\section{Off-line test}

We have successfully extracted a stable nickel ion-beam, evaporated from a nickel filament placed in the gas cell and ionized using the laser resonance ionization technique, in an off-line test. The ionization scheme of $3 d^{8} 4 s^{2} \quad(J=4) \rightarrow 3 d^{8} 4 s 4 p \quad(J=5)$ $\left(\lambda_{1}=232.074 \mathrm{~nm}\right.$ in vacuum $) \rightarrow$ auto ionization state $\left(\lambda_{2}=537.960 \mathrm{~nm}\right.$ in vacuum), as reported in Ref. [6], was employed in the present test. Using a nickel beam with an energy of $28 \mathrm{keV}$, we optimized the RF and DC voltages of the SPIG for cooling and 


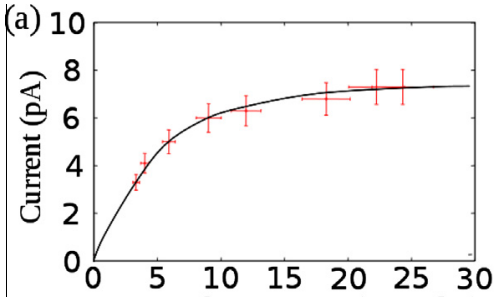

First step laser energy ( $\mu \mathrm{J} /$ pulse)

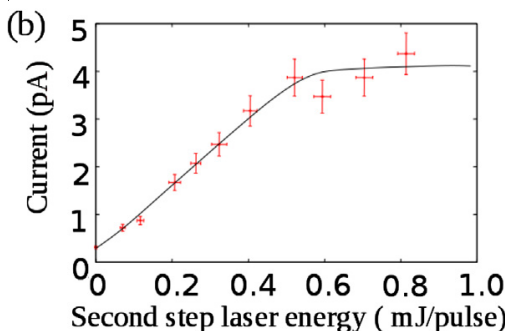

Fig. 4. Laser pulse energy dependence as a function of (a) first step and (b) second step laser intensities. Lines are provided as guides for the eyes. In (a), the laser wavelength and pulse energy of the second step laser were fixed to be $423.784 \mathrm{~nm}$ and $0.8 \mathrm{~mJ} /$ pulse. In (b), the laser wavelength and pulse energy of the first step laser were fixed to be $248.402 \mathrm{~nm}$ and $25 \mu \mathrm{J} /$ pulse.

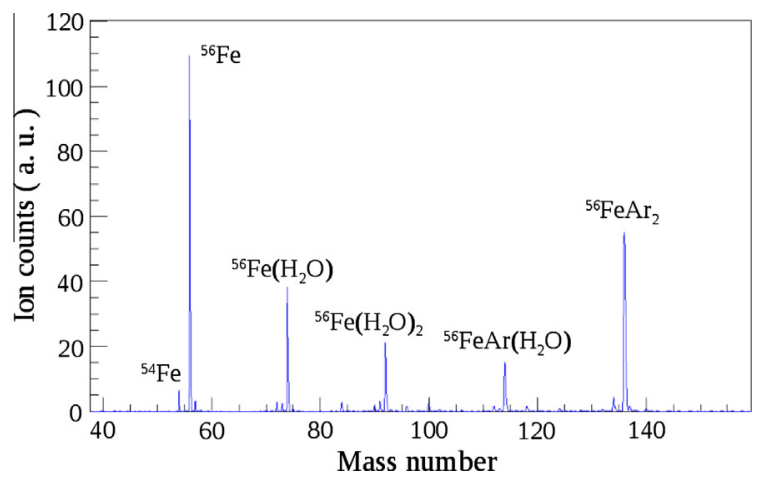

Fig. 5. Mass spectrum obtained using an iron filament with purified argon gas.

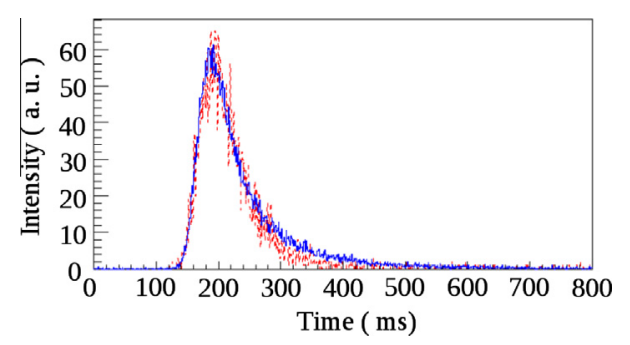

Fig. 6. Time profile of ${ }^{56} \mathrm{Fe}$ ionized at the primary beam position.

extracting the beam, and obtained a mass-resolving power of 900 , which is consistent with the design value [12] and is sufficient for selecting only ions having mass numbers of interest.

The absolute extraction efficiency and selectivity of KISS can be evaluated only by an on-line test to measure the beam intensities implanted in and extracted from the gas cell. In addition, plasma in the gas cell induced by the primary beam is reported to reduce the ionization efficiency and selectivity [8]. In order to measure the absolute extraction efficiency and selectivity under the plasma, it is necessary to perform an on-line test and measure the efficiency and selectivity as a function of the primary beam intensity.
As preparation for the on-line test using ${ }^{56} \mathrm{Fe}$, we performed an off-line test using an iron filament to determine an ionization scheme for iron and to measure the mass distribution and transport time-profile for argon gas flow in order to determine the basic performance of the gas cell.

We searched for a more efficient ionization scheme for iron than that reported in Ref. [16]. The ionization potential of an iron atom is $7.90 \mathrm{eV} .{ }^{56} \mathrm{Fe}$ atoms vaporized from an iron filament placed in the gas cell were ionized by the laser resonance ionization technique and were extracted as a beam. The beam current was measured by a Faraday cup. A new ionization scheme involving the transition $3 d^{6} 4 s^{2}$ $(J=4) \rightarrow 3 d^{6} 4 s 4 p(J=5)\left(\lambda_{1}=248.402 \mathrm{~nm}\right.$ in vacuum $) \rightarrow$ auto ionization state $\left(\lambda_{2}=423.784 \mathrm{~nm}\right.$ in vacuum) was identified as being potentially more efficient. The saturation powers for the first and second step lasers $\left(\lambda_{1}, \lambda_{2}\right)$ were measured, as shown in Fig. 4. Only $20 \mu \mathrm{J} /$ pulse and $0.8 \mathrm{~mJ} /$ pulse for the first step and second step lasers, respectively, were required for the saturation, which were a factor of three lower than those for the ionization scheme in Ref. [16]. Here, the laser spot sizes were $8-10 \mathrm{~mm}$ in diameter.

Using an iron filament, we measured the mass-distribution in the region of $A=40-160$ in the off-line test, as shown in Fig. 5. It was found that the intensities of the iron isotopes with mass numbers of $A=54,56,57$ and 58 were consistent with the natural abundances of $5.8 \%, 91.8 \%, 2.1 \%$ and $0.28 \%$, respectively. About $40 \%$ of the iron formed hydrates such as Fe $\left(\mathrm{H}_{2} \mathrm{O}\right)_{n}(n=1$ and 2$)$ and $\mathrm{FeAr}\left(\mathrm{H}_{2} \mathrm{O}\right)$. In order to reduce the formation of hydrates and increase the extraction efficiency, it might be necessary to cool the gas cell and argon gas down to $\sim 100 \mathrm{~K}$ to freeze water molecules [18]. The design of a cryogenic gas cell is in progress.

Fig. 6 shows a time profile of ${ }^{56} \mathrm{Fe}$ ionized at the primary beam position. A single shot of the ionization lasers were delivered at time $t=0 \mathrm{~ms}$ and the time profile was measured. The time profile shows the extraction time profile from the gas cell. The solid (blue) and dashed (red) lines are the measured and simulated time profiles of ${ }^{56} \mathrm{Fe}$, respectively. The simulated profile is in good agreement with the measured profile. This allows us to estimate the mean extraction time to be about $190 \mathrm{~ms}$, which is shorter than the expected lifetime $\left(t_{1 / 2}=423 \mathrm{~ms}\right)$ for ${ }^{200} \mathrm{~W}$.

\section{Summary}

We constructed the KEK Isotope Separation System (KISS) at RIKEN to study the $\beta$-decay properties of neutron-rich isotopes with neutron numbers around $N=126$ for applications in astrophysics. Using nickel and iron atoms evaporated from filaments, an off-line performance test of the gas cell system and the KISS was successfully performed. We established a new and efficient ionization scheme for iron for use in on-line tests. The extraction efficiency and the selectivity of KISS will be further investigated by on-line tests.

\section{Acknowledgments}

The authors acknowledge the staff of the RIKEN accelerator for their support. This work has been supported by Grant-in-Aids for Scientific Research (A) (S.C. Jeong, Grant No. 23244060) and for young scientists (B) (Y. Hirayama, Grant No. 24740180) from the Japan Society for the Promotion of Science (JSPS), by FWO-Vlaanderen (Belgium), by GOA/2010/010 (BOF KU Leuven), and by a grant from the European Research Council (ERC-2011-AdG-291561-HELIOS).

\section{References}

[1] E.M. Burbidge et al., Rev. Mod. Phys. 29 (1957) 547.

[2] S.C. Jeong et al., KEK, Report 2010-2 (2010).

[3] C.H. Dasso et al., Phys. Rev. Lett. 73 (1994) 1907.

[4] A. Winther, Nucl. Phys. A572 (1994) 191; A. Winther, Nucl. Phys. A594 (1995) 203. 
[5] Y.X. Watanabe et al., these proceedings.

[6] Yu. Kudryavstev et al., Nucl. Instrum. Methods B 114 (1996) 350.

[7] Yu. Kudryavstev et al., Nucl. Instrum. Methods B 179 (2001) 412.

[8] M. Huyse et al., Nucl. Instrum. Methods B 187 (2002) 535.

[9] Yu. Kudryavstev et al., Nucl. Instrum. Methods B 204 (2003) 336.

[10] M. Facina et al., Nucl. Instrum. Methods B 226 (2004) 401.

[11] Yu. Kudryavstev et al., Nucl. Instrum. Methods B 267 (2009) 2908.

[12] H. Ishiyama et al., RIKEN Accele. Prog. Rep. 45 (2012) 151.
[13] H.J. Xu et al., Nucl. Instrum. Methods A 333 (1993) 274.

[14] Y. Hirayama et al., RIKEN Accele. Prog. Rep. 45 (2012) 152.

[15] J.F. Ziegler, Available from: <http://www.srim.org/>.

[16] T. Sonoda et al., Nucl. Instrum. Methods B 295 (2013) 1.

[17] T. Tachibana, M. Yamada, Proc. Inc. Conf. on Exotic Nuclei and Atomic Masses, Arles (1995) 763

[18] A. Saastamoinen et al., Nucl. Instrum. Methods A 685 (2012) 70. 